\title{
Análise do aprendizado em programação de estudantes do ensino técnico integrado do Instituto Federal de Pernambuco
}

\author{
Leonardo S. Silva ${ }^{1}$ \\ ${ }^{1}$ Coordenação de Informática - Instituto Federal de Pernambuco (IFPE) \\ CEP 55299-390 - Garanhuns - PE - Brazil \\ leonardo.silva@garanhuns. ifpe.edu.br
}

\begin{abstract}
Understanding the current educational context is necessary to promote improvements in programming learning. Statistical analysis were applied in academic data of 301 high school students enrolled in the course of introduction to programming in IFPE. Students performance is not influenced by gender or determinant to the choice of higher education courses in informatics. However, it has strong correlation with mathematical performance. The results contribute to an understanding of the subject, especially in the professional educational context.
\end{abstract}

Resumo. Compreender o contexto educacional é fundamental para promover melhorias no aprendizado de programação. Análises estatísticas foram aplicados aos dados acadêmicos de 301 estudantes do ensino médio matriculados na disciplina de introdução á programação do IFPE. Foi observado quue o desempenho dos estudantes não é influenciado pelo gênero e não determinou a escolha por cursos superiores na área de computação. Por outro lado, este desempenho possui forte correlação com o desempenho em matemática. Os resultados contribuem para uma compreensão sobre o tema em especial no contexto da educação profissional.

\section{Introdução}

O ensino de programação nos cursos técnicos integrados ao ensino médio dos Institutos Federais (IF) pode ser considerado pioneiro no Brasil. Por meio deles, jovens adquirem habilidades consideradas fundamentais no mundo digital atual e antes restritas ao ensino superior [Barr and Stephenson 2011].

Em razão da natureza complexa da disciplina, aprender a programar não é uma atividade simples e afeta diretamente os estudantes nos cursos técnicos integrados [Fernandes and Junior 2014]. Este estudo se insere neste contexto e tem por objetivo compreender, sob múltiplas observações, o desempenho dos estudantes que cursaram a disciplina de introdução à programação, do curso técnico em informática integrado ao ensino médio do Instituto Federal de Pernambuco.

As seguintes questões de pesquisa são investigadas neste trabalho: a) qual o desempenho dos estudantes matriculados no curso técnico de informática integrado ao ensino médio na disciplina de introdução a programação? b) existe associação entre a opção por cursos superiores na área de computação e o desempenho dos estudantes em programação? c) o gênero influencia na escolha dos estudantes por cursos superiores na área de computação? d) existe diferença entre gêneros no desempenho em programação? e) o desempenho dos estudantes em matemática está associado ao desempenho em programação?

Cálculos estatísticos foram realizados para responder às questões de pesquisa estabelecidas, e seus resultados indicam que: o índice de reprovação na disciplina é de 52,5\%; o desempenho dos estudantes e o seu gênero não são determinantes na opção por cursos superiores na área de computação; não foi observada associação estatística 
entre o gênero e o desempenho em programação. Por fim, este desempenho está correlacionado ao desempenho na disciplina de matemática.

\section{Trabalhos relacionados}

Diversos trabalhos buscaram compreender os resultados dos estudantes na disciplina de introdução à programação, em suas instituições.

Bosse and Gerosa (2015) observaram o desempenho de estudantes de programação em um contexto do ensino superior. Em média, os índices de reprovação foram de aproximadamente $30 \%$, consideravelmente inferior ao encontrado por este estudo $(52,5 \%)$.

Campos (2010) também coletou dados acadêmicos do aprendizado de programação de instituições de ensino de nível superior. O resultado encontrado aponta para um índice de reprovação médio de $60 \%$. Quantitativo próximo ao encontrado por este estudo.

Em um contexto educacional mais próximo, Fernandes and Junior (2014), investigaram dados acadêmicos dos estudantes do integrado matriculados em introdução à programação do IFC. Um índice de reprovação de $15 \%$ foi encontrado.

Em comparação aos trabalhos citados, este estudo buscou avançar nesta área ao analisar a relação do desempenho dos estudantes no aprendizado em programação com outras variáveis não abordadas pelos autores citados. Cita-se também como contribuição, a análise com dados de estudantes do IF e que possibilita uma discussão mais ampla com outros pesquisadores deste contexto educacional tão singular.

\section{Metodologia}

\subsection{Coleta dos dados}

Os dados dos estudantes utilizados neste estudo foram obtidos com o setor de registro acadêmico do campus Garanhuns do IFPE. Os seguintes dados foram solicitados: gênero (masculino ou feminino), resultados finais nas disciplinas de introdução à programação e matemática (aprovado ou reprovado) e suas médias finais (intervalo de 0 à 10). A opção por curso superior na área de computação (sim ou não), foi coletada em pesquisas na Internet, nos resultados publicados de vestibulares. Nos casos em que as informações não foram localizadas, entrou-se em contato com os estudantes. Quando isto não foi possível, removeu-se o estudante da amostragem utilizada no cálculo estatístico da questão e).

\subsection{Descrição da amostra}

Em razão dos questionamentos de pesquisa apresentados abordarem diferentes contextos, diferentes tamanhos de amostra foram utilizados.

Os cálculos estatísticos adotados para responder as questões a) e d) utilizaram uma amostragem de 301 estudantes. Este quantitativo representa todos os estudantes matriculados na instituição no curso técnico de informática integrado ao ensino médio no período de 2012 à 2017. Deste quantitativo, 171 eram do gênero masculino $(56,8 \%)$ e 130 do gênero feminino $(43,2 \%)$. Apenas foram considerados os resultados dos estudantes na primeira vez que cursaram a disciplina de introdução à programação, havendo reprovação, os resultados posteriores foram descartados.

Para os cálculos estatísticos das questões b) e c), utilizou-se uma amostra de 35 estudantes. Este foi o quantitativo de estudantes que não evadiu durante o curso, realizou o $4^{\circ}$ ano do ensino médio integrado e se inscreveu no vestibular.

Para avaliar a relação entre as notas em introdução à programação e matemática, 
da questão e), foram utilizados os dados de 106 estudantes pertencentes a 3 turmas dos anos 2013, 2015 e 2016. Estas turmas apresentaram os mesmos professores de introdução à programação e matemática. Desta forma, foi possível isolar este fator, uma vez que a mudança de professores, entre anos, poderia afetar o resultado.

\section{Resultados e análise}

\subsection{Questão a)}

De uma amostra de 301 estudantes que cursaram introdução à programação, 143 foram aprovados $(47,5 \%)$ e 158 reprovados $(52,5 \%)$. Como discutido na seção 2, observa-se uma grande variação de resultados quando comparados entre instituições. Compreender estas diferenças requer uma análise mais ampla sobre o contexto educacional, social e econômico de cada região, e suas influencias sobre o desempenho dos estudantes. Ainda sim, com todas as singularidades e diferenças, o índice encontrado para o campus Garanhuns pode ser considerado alto, o que demanda ações institucionais que visem sua redução.

Neste sentido, sugere-se como trabalho futuro analisar o cenário em que o IFC se insere, bem como investigar o seu contexto pedagógico e curricular, com o objetivo de identificar ações que possam ter contribuído para os índices desta instituição.

Além disto, sugere-se também como trabalho futuro contabilizar a quantidade de vezes que os estudantes cursam a disciplina de introdução à programação, considerando os dados dos reprovados. Como também, observar se existe correlação entre o resultado do estudante (aprovado sem reprovação ou aprovado com reprovação) no desempenho em outras disciplinas de períodos posteriores, como exemplo, em programação orientada a objetos.

\subsection{Questões b), c) e d)}

Para responder as questões b), c) e d), testes estatísticos foram realizados por meio do Qui-Quadrado de Pearson [McHugh 2013]. A opção por este teste ocorreu em razão do uso de variáveis nominais para representar as informações analisadas.

Não foi observada associação de dependência entre a opção dos estudantes por cursos na área de computação e o desempenho em programação $(p$-value $=0.55)$, questão b); não foi observada associação de dependência entre a opção dos estudantes por cursos na área de computação e o seu gênero ( $p$-value $=0.63)$, questão c); não foi observada associação de dependência entre o desempenho em programação e o gênero $(p$-value $=0.22)$, questão d).

Havia uma hipótese de que estudantes optariam no vestibular por formação superior na área de computação em razão do seu desempenho em introdução à programação. Isto poderia ser justificado pela afinidade com o tema, por exemplo. No entanto, com os resultados obtidos não é possível afirmar isto.

As observações sobre o gênero são bastante positivas. Pode-se considerar que a instituição e seus servidores estão ofertando um ambiente que oferece oportunidades e estímulos iguais para ambos os gêneros.

\subsection{Questão e)}

Para analisar a correlação entre as notas dos estudantes em introdução à programação com as suas notas em matemática, optou-se pelo uso do teste de Spearman em razão da ausência de normalidade dos dados. Esta ausência foi identificada por meio do teste de Shapiro-Wilk ( $p$-value <0.05).

Foi observada uma correlação forte e positiva de 0,76 entre as notas dos estudantes em introdução à programação e matemática. Isto corrobora as hipóteses de 
professores da área que justificam parte do desempenho no aprendizado em programação à falta de base matemática [Gomes and Mendes 2007].

Sugere-se como trabalho futuro observar o desempenho dos estudantes também em outras disciplinas, como inglês e português. Alguns estudos argumentam que estes conhecimentos podem influenciar no aprendizado de programação [Raabe 2016; Gomes and Mendes 2007].

\section{Conclusão}

O ensino de programação nos cursos técnicos integrados ao ensino médio representa uma grande oportunidade para que jovens possuam o primeiro contato com a área.

Compreender como a disciplina de introdução à programação se insere no contexto dos institutos federais é importante para promover melhorias. O objetivo deste trabalho é oferecer subsídio estatístico sobre diferentes variáveis e suas associações com o desempenho dos estudantes nesta disciplina.

Os resultados apontam para um índice de reprovação de 52,5\%, superior à média internacional. Além disto, observa-se que o desempenho na disciplina e opção por cursos superiores na área não são influenciados pelo gênero dos estudantes. Por fim, foi identificada uma forte correlação estatística entre o desempenho destes alunos na disciplina de introdução à programação e o desempenho na disciplina de matemática.

A compreensão sobre o contexto em que os estudantes do IFPE se inserem, contribui para que os servidores deste campus possam propor melhorias, como também possibilita a comparação com o resultado de outros Institutos Federais. Espera-se com isto, ampliar o conhecimento e debate sobre o ensino de programação em cursos técnicos.

\section{Referências}

Barr, V., and Stephenson, C. (2011). Bringing computational thinking to K-12: what is Involved and what is the role of the computer science education community?. Acm Inroads, 2(1), 48-54.

Bosse, Y., and Gerosa, M. A. (2015). Reprovações e Trancamentos nas Disciplinas de Introdução à Programação da Universidade de São Paulo: Um Estudo Preliminar. In WEI-Workshop sobre Educação em Computação.(2015) (pp. 1-10).

Campos, R. L. B. L. (2010). Metodologia ERM2C: Para melhoria do processo de ensino-aprendizagem de lógica de programação. In XVIII Workshop sobre Educação em Computação.

Fernandes, V., and Junior, V. F (2014). Linguagem de programação: evasão e reprovação no instituto federal catarinense. In IX Mostra Nacional de Iniciação Científca e Tecnológica Interdisciplinar.

Gomes, A., and Mendes, A. J. (2007). Learning to program-difculties and solutions. In International Conference on Engineering Education-ICEE (Vol. 2007).

McHugh, M. L. (2013). The chi-square test of independence. Biochemia medica: Biochemia medica, 23(2), 143-149.

Raabe, A. L. A., Santana, A. L. M., Zanini, A. S., \& Vieira, M. F. V. (2016). Influência dos enunciados na resolução de problemas de programação introdutória. Revista Brasileira de Informática na Educação, 24(1).

Watson, C., and Li, F. W. (2014). Failure rates in introductory programming revisited. In Proceedings of the 2014 conference on Innovation \& technology in computer science education (pp. 39-44). ACM. 